\title{
Diagnosis and Treatment Outcomes of Tuberculosis in Relation to Gender and HIV STATUS in South Benin
}

\author{
Ablo Prudence Wachinou ${ }^{1,2 *}$, Serge Ade1,3,4, Gildas Agodokpessi1,2, Berenice Awanou1, \\ Dissou Affolabi' ${ }^{1}$, Wilfried Bekou' ${ }^{1}$, Marius Esse ${ }^{1}$, Gabriel Ade ${ }^{1}$, \\ Severin Anagonou' ${ }^{1}$, Anthony D. Harries ${ }^{4,5}$
}

${ }^{1}$ Programme National contre la Tuberculose (PNT), Cotonou, Bénin

${ }^{2}$ Faculté des Sciences de la Santé (FSS), Université d'Abomey-Calavi, Cotonou, Bénin

${ }^{3}$ Faculté de Médecine de Parakou, Université de Parakou, Parakou, Bénin

${ }^{4}$ International Union against Tuberculosis and Lung Disease, Paris, France

${ }^{5}$ London School of Tropical Medicine and Hygiene, London, UK

Email: *wachinouprudence@yahoo.fr

How to cite this paper: Wachinou, A.P., Ade, S., Agodokpessi, G., Awanou, B., Affolabi, D., Bekou, W., Esse, M., Ade, G., Anagonou, S. and Harries, A.D. (2017) Diagnosis and Treatment Outcomes of Tuberculosis in Relation to Gender and HIV STATUS in South Benin. Journal of Tuberculosis Research, 5, 189-200. https://doi.org/10.4236/jtr.2017.53021

Received: August 4, 2017

Accepted: September 26, 2017

Published: September 29, 2017

Copyright $\odot 2017$ by authors and Scientific Research Publishing Inc. This work is licensed under the Creative Commons Attribution International License (CC BY 4.0).

http://creativecommons.org/licenses/by/4.0/

\begin{abstract}
Background: In Benin, little is known about the influence of both gender and HIV-status on diagnostic patterns and treatment outcomes of Tuberculosis (TB) patients. Objective: To assess whether differences in gender and HIV status affect diagnostic patterns and treatment outcomes of TB patients. Methods: Retrospective cohort study of patients registered in 2013 and 2014 in the three largest TB Basic Management Units in south Benin. Results: Of 2694 registered TB patients, 1700 (63.1\%) were male. Case notification rates were higher in males compared with females (96 vs 53/100,000 inhabitants). The male to female ratio was 1:1 in HIV positive patients, but was 2:1 among HIV negative cases. In HIV-positive patients, there were no differences in TB types between men and women. In HIV-negative patients, there were significantly higher proportions of females with clinically diagnosed pulmonary TB ( $\mathrm{p}=$ $0.04)$ and extrapulmonary TB $(\mathrm{p}<0.001)$. Retreatment TB was 4.65 times higher amongst males compared with females. For New bacteriologically confirmed pulmonary $\mathrm{TB}$, no differences were observed in treatment outcomes between genders in the HIV positive group; but significantly more unfavorable outcomes were reported among HIV negative males, with higher rates of failure $(\mathrm{p}<0.001)$ and loss-to-follow up $(\mathrm{p}=0.02)$. Conclusion: The study has shown that overall TB notification rates were higher in males than in females in south Benin, with more females co-infected with HIV. Unfavorable outcomes were more common in HIV-negative males.
\end{abstract}




\section{Keywords}

Tuberculosis, Diagnosis, Treatment Outcomes, Gender, HIV

\section{Introduction}

Tuberculosis (TB) has been a serious burden to mankind over many generations, and, despite major advances in TB control efforts in the last century, the disease remains a major global health concern [1]. Despite considerable resources allocated for disease control, millions of new cases are detected each year. For example, in 2015, the World Health Organization (WHO) estimated that there were 10.4 million new cases worldwide [2]. Low and middle income countries, many of which are located in sub-Saharan Africa, are the most affected, with the main contributing factors being extreme poverty and a high prevalence of $\mathrm{Hu}$ man Immunodeficiency Virus (HIV) infection [1] [2]. HIV is the most important risk factor for TB and is also known to negatively impact treatment outcomes of co-infected TBHIV patients [3] [4]. Women are more vulnerable at acquiring HIV infection compared with men [5], and therefore they are at higher risk of developing TB [4]. However, at a global level, active TB disease is more common in men according to annual WHO reports [2].

TB case notification rates among men and women differ in different settings, constituting one of the epidemiological curiosities of this disease. Despite much discussion in the scientific literature about the reasons for these disparities, it is still unclear why this happens [5]. Biological and behavioural differences, inequity in accessing health care and pregnancy have been identified as possible key reasons, although convincing associations have yet to be shown [6] [7]. It is possible that HIV-status plays an important part in determining gender-related differences in TB diagnosis and management, and this needs to be addressed. A better understanding of gender and HIV status may help the global community reach the "End TB Strategy" targets of reducing TB incidence and death by $90 \%$ and $95 \%$ respectively in 2030 compared with 2015 [8]. The WHO TB case notification reports, in their current format, do not allow such analyses on TB diagnosis patterns and outcomes with respect of both gender and HIV-status [2], but this is something that can be achieved at a programmatic level.

Benin is a West Africa country with a population of approximately 10 million inhabitants [9]. The country annually notifies between 39 - 41 cases/100,000 inhabitants [10]. Although the general population is predominantly female (51.2\%), notified TB cases are much higher among men [9] [10] [11]. Data on TB are reported using the same WHO notification forms, which are the basis of decision-making in the programme. An operational research study was therefore undertaken to investigate whether there were any significant differences in diagnostic patterns and treatment outcomes of TB in relation to both gender and HIV-status. 
Using data from patients registered in 2013 and 2014 in south Benin, specific objectives of the study were: 1 ) to determine TB case notifications among males and females; and 2) to compare between both genders, with respect to their HIV status: a) Male to Female Ratios (MFR); b) types of TB; and c) treatment outcomes.

\section{Materials and Methods}

Study design. This was a retrospective cohort study using routinely collected data with the data collected between January and March 2017.

\subsection{General Setting}

Benin shares borders with Nigeria, Togo, Niger and Burkina-Faso. It is a low income country with a gross national income per capita of US\$840 in 2015 [12]. The probability of dying between 15 and 60 years was 59 years in men and 61 years in women in 2015 [13]. HIV prevalence in the general population is $1.2 \%$. This prevalence is slightly higher among females compared with males (1.4\% vs $1.0 \%)$ [14]. Antiretroviral therapy coverage in those infected with HIV was similar in 2015 [15]. Regarding other risk factors for TB, diabetes mellitus prevalence in the country was $12.4 \%$ in one survey with no significant differences found between males and females (12.7\% vs 12.2\%) [16].In Benin, the prevalence of smoking is $9.5 \%$ in men and $0.5 \%$ in women [16].

\subsection{Study Sites}

The study was carried out at the country's three largest Basic Management Units (BMUs) out of the 19 that serve the southern region: "Centre National Hospitalier Universitaire de Pneumo-phtisiologie" (CNHUPP) at Cotonou, "Centre de Pneumo-phtisiologie d'Akron (CPP-Akron)" at Porto-Novo, and "Centre de Santé d Abomey-Calavi" (CS Calavi) at Abomey-Calavi. These three BMUs notify about $60 \%$ of all TB cases in the region and one third of all cases reported in the whole country [10]. The catchment population of the three BMUs was $1,822,391$, of whom $51.4 \%$ were females according to the 2013 national census [9].

\subsection{TB Diagnosis and Management in Benin}

A National TB Programme (NTP) under the Ministry of Health is responsible for implementing all TB control strategies at the different levels. The Programme follows the International Standards for Tuberculosis Care (ISTC) and WHO recommendations [17] [18]. Diagnosis and treatment are decentralized in 67 BMUs across the country and are provided free of charge regardless of the patient's gender or age.

\subsubsection{Diagnosis}

Pulmonary TB diagnosis. Routinely, a person coughing for more than 2 weeks is regarded as having presumptive pulmonary $\mathrm{TB}$. He/she is requested to provide 
two sputum samples for smear microscopy for acid-fast bacilli. The patient is classified as "Bacteriologically confirmed Pulmonary TB" (BCPT) if there is a positive result on at least one sputum specimen. He/she is then classified into a new or retreatment case. A new case is someone who has never been treated for TB or treated for less than one month; otherwise, that person is a retreatment case. Those in whom the TB diagnosis is not bacteriologically confirmed but who do not respond to broad spectrum antibiotics and are thought to have TB are diagnosed as clinically diagnosed pulmonary TB (CDPT).

Extrapulmonary TB diagnosis (EPT): the diagnosis of this type of TB is mostly empirical, based on focal symptoms and/or radiologic/histological/laboratory abnormalities.

\subsubsection{Treatment}

All new cases are treated with the same first line anti-tuberculosis treatment regimen. Patients receive daily ethambutol, rifampicin, isoniazid and pyrazinamide during two months (intensive phase), followed by four months of rifampicin and isoniazid (continuation phase). While treatment is directly observed during the intensive phase for all new BCPT cases, those who are clinically diagnosed or have EPTB are supplied every two weeks with anti-TB drugs for daily self- administration.

Outcomes of all treated TB patients are reported using the WHO standardized definitions: cured, treatment completed, death, failure, loss-to-follow up. Details on these terms are provided in Box 1 [18].

\subsubsection{TB-HIV Co-Infection}

All diagnosed TB cases are systematically offered HIV testing. In addition, for those who are found co-infected with HIV, appropriate care i.e. Cotrimoxazole Preventive Therapy (CPT) and Antiretroviral Therapy (ART) is provided in line with WHO recommendations [4].

\subsection{Patients}

All TB cases recorded in TB registers in CNHPP, CHPP Akron and CS Calavi in 2013 and 2014 were included in the study.

\subsection{Data Variables and Collection}

Data for this study were collected from paper-based TB registers and included: age, sex, types of TB, bacillary load at the time of diagnosis for new BCPT, sputum smear results at two months, treatment outcomes. These data were entered into an electronic file using EpiDataEntry 3.1 (EpiData Association, Odense, Denmark).To reduce errors, the electronic file was later cross-checked with TB registers by another data manager.

\subsection{Data Analysis}

Data were analyzed using Epidata AnalysisV2.2.2.182 (EpiData Association, 
Box 1. Definition of treatment outcomes for tuberculosis patients.

\begin{tabular}{ll}
\hline \multicolumn{1}{c}{ Outcome } & \multicolumn{1}{c}{ Definitions } \\
\hline Cured & $\begin{array}{l}\text { A pulmonary TB patient with bacteriologically confirmed TB at the } \\
\text { beginning of treatment who was smear- or culture-negative in the last } \\
\text { month of treatment and on at least one previous occasion. }\end{array}$ \\
& $\begin{array}{l}\text { A TB patient who completed treatment without evidence of failure BUT } \\
\text { with no record to show that sputum smear or culture results in the last } \\
\text { month of treatment and on at least one previous occasion were negative, } \\
\text { either because tests were not done or because results are unavailable. }\end{array}$ \\
Treatment completed & $\begin{array}{l}\text { A TB patient whose sputum smear or culture is positive at month } 5 \text { or later } \\
\text { during treatment. }\end{array}$ \\
Treatment failed & $\begin{array}{l}\text { A TB patient who dies for any reason before starting or during the course } \\
\text { of treatment. }\end{array}$ \\
Died & $\begin{array}{l}\text { A TB patient who did not start treatment or whose treatment was } \\
\text { interrupted for } 2 \text { consecutive months or more. }\end{array}$ \\
Lost to follow-up & $\begin{array}{l}\text { A TB patient for whom no treatment outcome is assigned. This includes } \\
\text { cases "transferred out" to another treatment unit as well as cases for } \\
\text { whom the treatment outcome is unknown to the reporting unit. }\end{array}$ \\
Not evaluated &
\end{tabular}

Odense, Denmark) and SPSS Statistics for Windows, Version 20.0 (Armonk, NY: IBM Corp). Continuous variables were appropriately described with means $( \pm$ Standard deviation) or medians ( \pm Interquartile range). Categorical data were described using percentages and proportions. Comparisons of categorical data were made between men and women using the chi-square test. Levels of significance were set at less than 0.05 .

\subsection{Ethics}

This study was approved by the National TB Programme. Because of its retrospective nature, approval of the local ethics committee was not required according to their recommendations. Ethics approval for writing up and publishing the data was obtained, however, from the Ethics Advisory Group, International Union against Tuberculosis and Lung Disease, Paris, France. Data were collected anonymously, and using a unique identifier. All databases were confidentially kept and were protected with a password, which was only accessible to authorized persons.

\section{Results}

In total, $2694 \mathrm{~TB}$ patients were registered in 2013 and 2014, of whom 1700 (63.1\%) were males and 994 (36.9\%) were females. The TB case notification rate 
was higher among males compared with females (96 vs 53/100 000 inhabitants; $\mathrm{p}<0.001)$. HIV prevalence was lower in males than females $(16.8 \%$ vs $26.8 \%$; $\mathrm{p}<0.001)$.

\subsection{Demographic Characteristics}

Stratification of MFR by HIV status and age groups is shown in Table 1. In general, males were older than females $(36.2 \pm 13.4$ vs $32.4 \pm 14.1 ; \mathrm{p}<0.001)$. Large variations of MFR were observed across age groups in both HIV positive and negative patients. However, in general, while notified TB cases were similar in males and females in those diagnosed HIV positive, two men for one woman were reported among HIV negative cases. In the modal age group (25 - 34 years) which accounted for approximately one third of all cases, this ratio of MFR 2:1 among HIV negative patients was still observed, although the ratio reversed among HIV-negative patients. The most striking MFR differences were found in the 45 - 54 year age group, regardless of HIV-status.

\subsection{Diagnosis, Follow-Up and Treatment Outcomes}

Patients were stratified according to their HIV status, types of TB and gender; and results are presented in Table 2. In co-infected TBHIV cases, no significant differences were found between males and females with respect to the type of TB. In HIV negative patients, the proportions of females among new CDPT ( $\mathrm{p}=$ $0.04)$ and EPT $(\mathrm{p}<0.001)$ cases were significantly higher than males. Conversely, the number of males registered with retreatment TB was 4.65 times higher compared with females.

In all new BCPT patients, while the proportion of males with high baseline bacillary load (i.e., $>10$ acid fast bacilli /field on Ziehl-Neelsen staining with

Table 1. Stratification of Male to Female Ratio by HIV status and age groups of TB patients at CNHPP, CHPP Akron and CS Calavi in Benin, 2013-2014 ( $\mathrm{n}=2694)$.

\begin{tabular}{ccccc}
\hline Age groups (years) & $\mathrm{n}(\%)$ & All patients & HIV positive & HIV negative \\
\hline $0-4$ & $29(1.1)$ & $1.2: 1$ & $2.0: 1$ & $0.9: 1$ \\
$5-14$ & $81(3.0)$ & $0.9: 1$ & $0.7: 1$ & $1.0: 1$ \\
$15-24$ & $488(18.1)$ & $1.1: 1$ & $0.5: 1$ & $1.2: 1$ \\
$25-34$ & $835(31.0)$ & $1.5: 1$ & $0.5: 1$ & $2.0: 1$ \\
$35-44$ & $639(23.7)$ & $2.2: 1$ & $1.7: 1$ & $2.5: 1$ \\
$45-54$ & $370(13.7)$ & $3.2: 1$ & $2.3: 1$ & $3.6: 1$ \\
$55-64$ & $159(5.9)$ & $2.2: 1$ & $1.3: 1$ & $2.5: 1$ \\
$\geq 65$ & $93(3.5)$ & $1.5: 1$ & $1.0: 1$ & $1.6: 1$ \\
Total & $2694(100 \%)$ & $1.7: 1$ & $1.1: 1$ & $2.0: 1$ \\
\hline
\end{tabular}

CNHPP = "Centre National Hospitalier de Pneumo-Phtisiologie"; CHPP Akron = "Centre Hospitalier de Pneumo-Phtisiologie d'Akron"; CS Calavi = "Centre de Santé d'Abomey-Calavi"; HIV = Human Immunodeficiency Virus. 
Table 2. Distribution of patients by HIV status, type of TB and gender in CNHPP, CHPP Akron and CS Calavi, Benin, 2013-2014 (n = 2694).

\begin{tabular}{ccccc}
\hline & Male n $\left(\%{ }^{\mathrm{a}}\right)$ & Female n (\%) & MFR & p (value $)^{\mathrm{b}}$ \\
\hline HIV positive & 285 & 266 & 1.07 & - \\
New BCPT & $168(58.9)$ & $156(58.6)$ & 1.08 & 0.942 \\
New CDPT & $57(20.0)$ & $64(24.1)$ & 0.89 & 0.250 \\
EPTB & $34(11.9)$ & $32(12.0)$ & 1.06 & 0.917 \\
Retreatment TB & $26(9.1)$ & $14(5.3)$ & 1.86 & 0.081 \\
HIV negative & 1407 & 721 & 1.95 & - \\
New BCPT & $1082(76.9)$ & $534(74.1)$ & 2.03 & 0.147 \\
New CDPT & $80(5.7)$ & $58(8.0)$ & 1.38 & 0.04 \\
EPTB & $124(8.8)$ & $103(14.3)$ & 1.20 & $<0.001$ \\
Retreatment TB & $121(8.6)$ & $26(3.6)$ & 4.65 & $<0.001$ \\
\hline
\end{tabular}

CNHPP = "Centre National Hospitalier de Pneumo-Phtisiologie"; CHPP Akron = "Centre Hospitalier de Pneumo-Phtisiologie d'Akron"; CS Calavi = "Centre de Santé d'Abomey-Calavi” TB = Tuberculosis; BCPT = Bacteriologically Confirmed Pulmonary Tuberculosis; CDPT = Clinically Diagnosed Pulmonary Tuber-

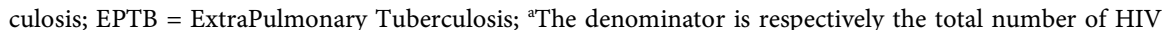
positive and HIV negative cases; ${ }^{\mathrm{b}}$ Comparisons were made between males and females in each type of tuberculosis.

light microscopy) was higher than in females $(27.8 \%$ vs $23.1 \%$; $\mathrm{p}=0.02)$, no difference was found after two months of treatment with respect to sputum smear conversion between the two groups $(83.8 \%$ vs $85.5 \%$; $\mathrm{p}=0.31)$.

Final treatment outcomes were stratified by gender and HIV-status and are shown in Tables 3(a)-(d). For New BCPT, whilst no differences in outcomes regarding gender were observed in HIV positive patients, significantly more males were found to have failed treatment $(\mathrm{p}<0.001)$ or were lost-to-follow up $(\mathrm{p}=0.02)$ in HIV negative cases (Table $3(\mathrm{a})$ ). In HIV positive EPT cases, there were more deaths ( $14.7 \%$ Vs $0 \%)$ and higher losses-to-follow up (11.8\% vs 6.5\%) among males (Table $3(\mathrm{c})$ ). In new CDPT and retreatment TB, no differences in treatment outcomes were found between males and females with respect to their HIV status (Table 3(b) and Table 3(d)). Finally, ART prescription for TBHIV patients was similar in both groups ( $86.3 \%$ for males vs $87.9 \%$ for females).

\section{Discussion}

This is the first study implemented in Benin that has assessed whether there were any differences in diagnosis and treatment outcomes between males and females with respect to their HIV-status.

The strengths of the study were that it was representative of the southern region of the country and large numbers of consecutively registered patients over a period of two years were included. The routine data were of high quality and produced by well trained health workers. Moreover, the data had been previously validated during quarterly supervisions and were reliable, as demonstrated in 
Table 3. Treatment outcomes stratified by HIV status and gender in CNHPP, CHPP Akron and CS Calavi, Benin, 2013-2014. (a) New Bacteriologically Confirmed Pulmonary Tuberculosis patients; (b) New clinically diagnosed pulmonary tuberculosis patients; (c) Extrapulmonary Tuberculosis patients; (d) Retreatment tuberculosis patients.

(a)

\begin{tabular}{cccc}
\hline & Male n (\%) & Female n (\%) & $\mathrm{p}(\text { value })^{\mathrm{a}}$ \\
\hline HIV positive & 279 & 262 & - \\
Successful outcome & $213(76.3)$ & $211(80.5)$ & 0.23 \\
Unsuccessful outcome & $66(23.7)$ & $51(19.5)$ & \\
Failure & $4(1.4)$ & $7(2.7)$ & 0.31 \\
Death & $42(15.1)$ & $33(12.6)$ & 0.41 \\
Loss-to-follow up & $20(7.2)$ & $11(4.2)$ & 0.14 \\
HIV negative & 1397 & 717 & - \\
Successful outcome & $1257(90.0)$ & $678(94.6)$ & $<0.001$ \\
Unsuccessful outcome & $140(10.0)$ & $39(5.4)$ & \\
Failure & $44(3.1)$ & $6(0.8)$ & $<0.001$ \\
Death & $46(3.3)$ & $20(2.8)$ & 0.53 \\
Loss-to-follow up & $50(3.6)$ & $13(1.8)$ & 0.02 \\
\hline
\end{tabular}

$\mathrm{HIV}=$ Human Immunodeficiency Virus; ${ }^{\mathrm{a} C o m p a r i s o n s ~ w e r e ~ m a d e ~ b e t w e e n ~ m a l e s ~ a n d ~ f e m a l e s ~ a m o n g ~ i n ~}$ each category of treatment outcome.

(b)

\begin{tabular}{cccc}
\hline & Male n (\%) & Female n (\%) & p (value) \\
\hline HIV positive & 57 & 64 & - \\
Successful outcome & $39(68.4)$ & $49(76.6)$ & 0.315 \\
Unsuccessful outcome & $18(31.6)$ & $15(23.4)$ & - \\
Death & $10(55.6)$ & $9(60.0)$ & 0.797 \\
Loss-to-follow up & $8(44.4)$ & $6(40.0)$ & - \\
HIV negative* & 78 & 58 & - \\
Successful outcome & $70(89.7)$ & $49(84.5)$ & 0.358 \\
Unsuccessful outcome & $8(10.3)$ & $9(23.4)$ & \\
Death & $4(50.0)$ & $3(33.3)$ & $0.838^{\varsigma}$ \\
Loss-to-follow up & $4(50.0)$ & $6(66.7)$ & \\
\hline
\end{tabular}

*02 males were "transferred out"; ${ }^{\circledR}$ The Fisher exact test was used; HIV = Human Immunodeficiency Virus.

(c)

\begin{tabular}{cccc}
\hline & Male n (\%) & Female n (\%) & p (value) \\
\hline HIV positive & 34 & 31 & - \\
Successful outcome & $25(73.5)$ & $29(93.5)$ & 0.031 \\
Unsuccessful outcome & $9(26.5)$ & $2(6.5)$ & - \\
Death & $5(14.7)$ & $0(0.0)$ & 0.545 \\
Loss-to-follow up & $4(11.8)$ & $2(6.5)$ & - \\
HIV negative & 123 & 103 & - \\
Successful outcome & $113(91.9)$ & $97(94.2)$ & 0.501 \\
Unsuccessful outcome & $10(8.1)$ & $6(5.8)$ & - \\
Death & $4(3.2)$ & $3(2.9)$ & $>0.999$ \\
Loss-to-follow up & $6(4.9)$ & $3(2.9)$ & -
\end{tabular}

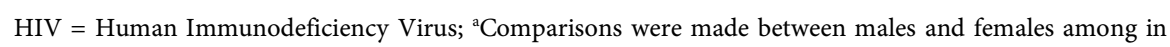
each category of treatment outcome. 
(d)

\begin{tabular}{cccc}
\hline & Male n (\%) & Female n (\%) & $\mathrm{p} \mathrm{(value)}^{\mathrm{a}}$ \\
\hline HIV positive & 24 & 13 & - \\
Successful outcome & $21(87.5)$ & $10(76.9)$ & 0.695 \\
Unsuccessful outcome & $3(12.5)$ & $3(23.1)$ & \\
Failure & $0(0.0)$ & $1(7.7)$ & - \\
Death & $3(12.5)$ & $2(15.4)$ & $>0.999$ \\
Loss-to-follow up & $0(0.0)$ & $0(0.0)$ & - \\
HIV negative & 121 & 25 & - \\
Successful outcome & $99(81.8)$ & $22(88.0)$ & 0.675 \\
Unsuccessful outcome & $22(18.2)$ & $3(12.0)$ & \\
Failure & $8(6.6)$ & $3(12.0)$ & 0.568 \\
Death & $6(5.0)$ & $0(0.0)$ & - \\
Loss-to-follow up & $8(6.6)$ & $0(0.0)$ & - \\
\hline
\end{tabular}

HIV = Human Immunodeficiency Virus; ${ }^{\mathrm{a} C o m p a r i s o n s ~ w e r e ~ m a d e ~ b e t w e e n ~ m a l e s ~ a n d ~ f e m a l e s ~ a m o n g ~ i n ~}$ each category of treatment outcome.

a study in 2010 [19]. The current study is reported according to the Strengthening the Reporting of Observational Studies in Epidemiology (STROBE) guidelines [20]. Limitations include its retrospective nature which does not allow any other validation apart from cross-checking with registers. Findings from this study also cannot be generalized to the whole country as the MFR ratio in relation with TB and HIV may vary across the regions.

One of the main findings was that male gender appeared to be a risk factor of developing TB, with a notified MFR in the population and a notified MFR in HIV negative cases that was 1.8 and 2 times higher respectively compared to females. This finding was similar to that reported by Gninafon et al. six years earlier in a nationwide survey [11] and also in 2016 in the WHO TB report [2], showing its consistency over the years. Elsewhere, many studies have also reported on the predominance of males in TB cases [1] [7] [21] [22] [23]. Several reasons for this observation have been previously discussed although not clearly confirmed. They include genuine biological differences between men and women (men seem to be more vulnerable to M. tuberculosis) [24] and a low access of females to education, information and also health care services due to social, economic and cultural barriers [7] [22]. In Benin, we think that rural to urban migration which mostly affects young men might play a role. These migrants are usually compelled to live in overcrowded urban dwellings that offer ideal conditions for tubercle bacilli to spread and disseminate. The pre- dominance of young men among TB patients in this study is in line with this assumption. In addition, smoking, which is more common among men in the country [16], may also partly contribute to the predominance of males. For example in one study, smoking was found to be a significant predictor that explains $33 \%$ of the variance in the MFR of TB notifications [25]. There may be other reasons to explain this situation. 
Not surprisingly, HIV prevalence that is higher in females in the general population, [14] was also found to be higher among TB patients. This finding is in agreement with reports from other settings in sub-Saharan Africa [3] [26] [27]. In terms of types of $\mathrm{TB}$, among HIV negative patients, women were more likely to be diagnosed with new CDPT and EPT than men and this in line with previous studies published in the country [3] [28] and also from elsewhere [23] [26] [27] [29] [30].

Interestingly, HIV positive patients, who also had a good ART coverage, had a satisfactory treatment success, which did not differ between males and females for all new pulmonary TB cases, although there needs to be improvement for HIV positive men who are diagnosed with EPT.

We were surprised and concerned about the unfavorable treatment outcomes, with more treatment failures and losses-to-follow up among men who were HIV negative and diagnosed with new BCPT. Since the two months sputum smear conversion rate was high and comparable in both men and women, we were expecting to find similar treatment success rates. In the scientific literature, men are usually reported as having a higher unsuccessful treatment rate, but different to our study, the findings were not analyzed with respect to patients' HIV-status [7] [21].

This study has some implications for the TB programme in this region of the country. First, more attention should be paid to men during the course of treatment, especially those who are diagnosed with new BCPT but are not infected by HIV. They should be advised on the importance of maintaining adherence during the continuation phase to avoid treatment failure and loss-to- follow up. For these patients, early tracing if required should also be initiated if they fail to appear on time at scheduled appointments. Second, men are more than four times more likely to have retreatment TB compared with women. More vigilance and information about previous $\mathrm{TB}$ is therefore needed whenever a man is diagnosed with TB in order to avoid misclassification and inadequate treatment administration. Third, reasons why males are more vulnerable to active TB than women needs to be investigated in this setting by qualitative and quantitative studies.

\section{Conclusion}

The study has shown that the TB notification rate was higher in males than in females in the southern region of Benin. Female TB patients were more likely to be HIV-positive. Unfavorable treatment outcomes were more common in HIV negative males. Further studies are needed to explain the country specific underlying reasons for these gender differences in TB case notifications in Benin and to assess if there are any variations across the different regions.

\section{Acknowledgements}

The authors thank all the health workers involved in TB care in the whole country, especially those in the southern region for their great job. 


\section{References}

[1] Maher, D. and Raviglione, M. (2006) Global Epidemiology of Tuberculosis. Clinics in Chest Medicine, 2, 952-953.

[2] World Health Organization (2016) Global Tuberculosis Report 2016. WHO/HTM/ TB/2016.13. WHO, Geneva. http://apps.who.int/iris/bitstream/10665/250441/1/9789241565394-eng.pdf?ua=1

[3] Agodokpessi, G., Ade, G., Ade, S., Wachinou, A.P., Affolabi, D., Anagonou, S. and Gninafon, M. (2012) Management of Tuberculosis and HIV Co-Infection in Cotonou, Benin. Médecine et Maladies Infectieuses, 42, 561-566. https://doi.org/10.1016/j.medmal.2012.07.012

[4] World Health Organization (2012) WHO Policy on Collaborative TB/HIV Activities Guidelines for National Programmes and Other Stakeholders.

http://apps.who.int/iris/bitstream/10665/44789/1/9789241503006_eng.pdf?ua=1\&ua $=1$

[5] UNAIDS (2016) Global AIDS Report 2016. Geneva. http://www.unaids.org/en/resources/documents/2016/Global-AIDS-update-2016

[6] Horton, K.C., Mac Pherson, P., Houben, R.M.G.J., White, R.G. and Corbett, E.L. (2016) Sex Differences in Tuberculosis Burden and Notifications in Lowand-Middle-Incomes Countries: A Systematic Review and Meta-Analysis. PLoS Medicine, 13, 1-23. https://doi.org/10.1371/journal.pmed.1002119

[7] Thorson, A., Long, N.H., Johanson, E. and Diwan, V.K. (2010) Tuberculosis and Gender. Bras Pneumol publicaçao of da Soc Bras Pneumol e Tisilogia, 36, 621-625.

[8] World Health Organization (2015) The End TB Strategy. World Health Organization, Geneva. http://www.who.int/tb/post2015_strategy/en/

[9] Institut National de la Statistique et de l'Analyse Economique (INSAE) (2015) RGPH4: Que retenir des effectifs de population? Cotonou. https://www.google.bj/?gws_rd=cr\&ei=SNntWMvfEcn6aM_NpMgL\#q=[9]\%09Insti tut+National+de+la+Statistique + et + de $+1 \%$ E2\%80\%99Analyse+Economique+(INS AE).+RGPH4+:+Que+retenir+des+effectifs+de+population+?+Cotonou.2015

[10] World Health Organization (2016) Tuberculosis Country Profiles: Benin. World Health Organization, Geneva.

https://extranet.who.int/sree/Reports?op=Replet\&name=\%2FWHO_HQ_Reports\% 2FG2\%2FPROD\%2FEXT\%2FTBCountryProfile\&ISO2=BJ\&LAN=EN\&outtype=pdf

[11] Gninafon, M., Trebucq, A. and Rieder, H.L. (2011) Epidemiology of Tuberculosis in Benin. International Journal of Tuberculosis and Lung Disease, 15, 61-66.

[12] World Bank Data. Benin. http://data.worldbank.org/country/benin?view=chart

[13] World Health Organization (2000) Global Health Observatory Country Views. Benin. Statistics Summary. http://apps.who.int/gho/data/node.country.country-BEN

[14] Institut National de la Statistique et de l'Analyse Economique (INSAE) et ICF International (2013) Enquête Démographique et de Santé du Bénin 2011-2012. INSAE et ICF International, Calverton. http://www.dhsprogram.com/pubs/pdf/FR270/FR270.pdf

[15] Diabaté, S., Zannou, D.M., Geraldo, N., Chamberland, A., Akakpo, J., et al. (2011) Antiretroviral Therapy among HIV-1 Infected Female Sex Workers in Benin: A Comparative Study with Patients from the General Population. WJA, 1, 94-99. https://doi.org/10.4236/wja.2011.13014

[16] World Health Organization (2015) Benin enquête STEPS. http://www.who.int/chp/steps/Benin_2015_STEPS_FactSheet_FR.pdf 
[17] TB CARE I (2014) International Standards for Tuberculosis Care. 3rd Edition, The Hague. http://www.who.int/tb/publications/ISTC_3rdEd.pdf

[18] World Health Organization (2014) Definitions and Reporting Framework for Tuberculosis-2013 Revision. http://apps.who.int/iris/bitstream/10665/79199/1/9789241505345_eng.pdf

[19] Trebucq, A., Zellweger, J.P., Kassa, F., Lambregts, K. and Gninafon, M. (2010) Are the Statistical Data from Benin's National Tuberculosis Programme Reliable? International Journal of Tuberculosis and Lung Disease, 14, 434-439.

[20] Von Elm, E., Altman, D.G. and Egger, M. (2007) The Strengthening the Reporting of Observational Studies in Epidemiology (STROBE) Statement: Guidelines for Reporting Observational Studies. Bulletin of the World Health Organization, 85, 867-872. https://doi.org/10.2471/BLT.07.045120

[21] Mukherjee, A., Sarkar, A., Saha, I. and Chowdhury, R. (2012) Gender Differences in Notification Rates, Clinical Forms and Treatment Outcome of Tuberculosis Patients under the RNTCP. Lung India, 29, 120-122.

https://doi.org/10.4103/0970-2113.95302

[22] Van den Hof, S., Najilis, C.A., Bloss, E. and Straetemans, M. (2010) A Systematic Review on the Role of Gender in Tuberculosis Control. https://www.kncvtbc.org/uploaded/2015/09/Role_of_Gender_in_TB_Control.pdf

[23] Effiong, J.O. and Nwakaego, I.F. (2015) Gender Differences among Clients Attending Tuberculosis Unit of a Teaching Hospital in Southern Nigeria. Scholars Journal of Applied Medical Sciences, 3, 228-233.

[24] Neyrolles, O. and Quintana-Murci, L. (2009) Sexual Inequality in Tuberculosis. PLOS Medicine, 6, e1000199. https://doi.org/10.1371/journal.pmed.1000199

[25] Watkins, R.E. and Plant, A.J. (2006) Does Smoking Explain Sex Differences in the Global Tuberculosis Epidemic? Epidemiology \& Infection, 134, 333-339. https://doi.org/10.1017/S0950268805005042

[26] Lawson, L., Lawson, J.O., Olajide, I., Emenyonu, N., Bello, C.S.S., Olatunji, O.O., et al. (2008) Sex Differences in the Clinical Presentation of Urban Nigeria Patients with Pulmonary Tuberculosis. West African Journal of Medicine, 27, 82-86.

[27] Chanda-Kapata, P., Kapata, N., Klinkenberg, E., Grobusch, M.P. and Cobelens, F. (2017) The Prevalence of HIV among Adults with Pulmonary TB at a Population Level in Zambia. BMC Infectious Diseases, 17, 236. https://doi.org/10.1186/s12879-017-2345-5

[28] Ade, S., Harries, A.D., Trebucq, A., Ade, G., Agodokopessi, G., Adjonou, C., et al. (2014) National Profile and Treatment Outcomes of Patients with Extrapulmonary Tuberculosis in Bénin. PLoS ONE, 9, e95603. https://doi.org/10.1371/journal.pone.0095603

[29] Jaryal, A., Raina, R., Sarkar, M. and Sharma, A. (2011) Manifestations of Tuberculosis in HIV/AIDS Patients and Its Relationship with CD4 Count. Lung India, 28, 263-266. https://doi.org/10.4103/0970-2113.85687

[30] Mekonnen, D., Derbie, A. and Desalegn, E. (2015) TB/HIV Co-Infections and Associated Factors among Patients on Directly Observed Treatments Short Course in Northeastern Ethiopia: A 4 Years Retrospective Study. BMC Research Notes, 8, 666. https://doi.org/10.1186/s13104-015-1664-0 
Submit or recommend next manuscript to SCIRP and we will provide best service for you:

Accepting pre-submission inquiries through Email, Facebook, LinkedIn, Twitter, etc. A wide selection of journals (inclusive of 9 subjects, more than 200 journals)

Providing 24-hour high-quality service

User-friendly online submission system

Fair and swift peer-review system

Efficient typesetting and proofreading procedure

Display of the result of downloads and visits, as well as the number of cited articles Maximum dissemination of your research work

Submit your manuscript at: http://papersubmission.scirp.org/

Or contact 迆@scirp.org 